\title{
On homotopies of morphisms and admissible mappings
}

\author{
Zdzisław Dzedzej® and Tomasz Gzella
}

To the memory of A. Granas.

\begin{abstract}
The notion of homotopy in the category of morphisms introduced by Górniewicz and Granas is proved to be equivalence relation which was not clear for years. Some simple properties are proved and a coincidence point index is described.
\end{abstract}

Mathematics Subject Classification. Primary 55M20; Secondary 47H04, $54 \mathrm{C} 60$.

Keywords. Homotopy, morphisms, coincidence index, admissible mapping.

\section{Introduction}

In [9] Górniewicz and Granas defined a convenient category which allows to treat fixed point problems for a broad class of multivalued maps as coincidence problems for single-valued maps. Their notion of morphisms is still the object of interest in various aspects (see, e.g., [1,12,16-18,20,21]). The aim of this paper is to define a homotopy notion and to check its basic properties in their category. In particular, we prove the transitivity property of the homotopy what seems new (comp. the remark before Lemma 3.4. in [1, p. 602]). Note that in some papers, a different notion of homotopy is considered $[8,13]$. We prove some basic properties of the homotopy and also introduce a homotopy invariant - coincidence point index. This yields some known theorems on coincidence and fixed points (comp., e.g., [5,7,9,14,19,24]).

The index was in fact defined in [5] for a broader class of multivalued maps but then it appeared to be a set of indices. Note that our notion of homotopy appears to be not the usual one in the case of single-valued maps. It seems to be closer to the homology relation. Nevertheless, it is satisfactory from the point of view of index theory. 


\section{The Górniewicz-Granas category of morphisms}

By a space, we understand a Hausdorff topological space and by a map - a continuous transformation.

Definition 2.1. A map $p: X \rightarrow Y$ is a Vietoris map provided

1. $p$ is onto and closed (an image of a closed set is a closed set),

2. for each $y \in Y p^{-1}(y)$ is a compact acyclic set (i.e., all its reduced Čech homology groups with rational coefficients are zero).

We will denote Vietoris maps by a double arrow $p: X \Longrightarrow Y$. We make use of the following basic properties of them. Observe that Vietoris maps are proper maps (i.e., the preimage of a compact set is compact).

Theorem 2.2. (see [11, Ch. VI $\S 19$ Thm 3.2]) If $p: X \Longrightarrow Y$ then the induced linear map $p_{*}: H_{*}(X) \rightarrow H_{*}(Y)$ is an isomorphism. The composite of Vietoris maps is also a Vietoris map. The pullback of a Vietoris map is a Vietoris map. after.

We use the Čech homology theory with compact carriers here and there-

Given two spaces $X$ and $Y$, we consider the set of all diagrams of the form $X \stackrel{p}{\Longleftarrow} Z \stackrel{q}{\longrightarrow} Y$. Two such diagrams are said to be equivalent if there are maps $f: Z \rightarrow Z^{\prime}$ and $g: Z^{\prime} \rightarrow Z$ such that the following two diagrams commute
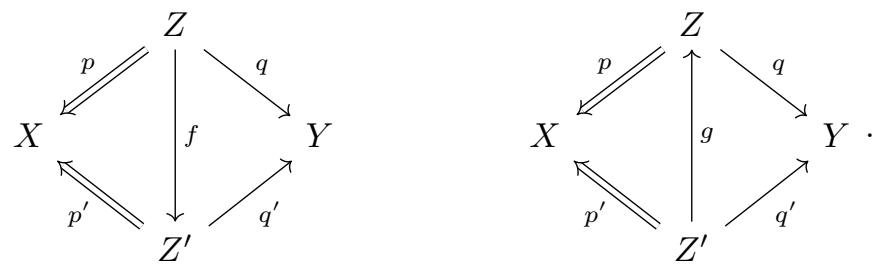

Clearly, this is an equivalence relation (see [9]). Let us note that in $[8,13]$ a different (more restrictive) relation is considered. The maps $f, g$ are required to be homeomorphisms there.

Definition 2.3. ([9]) An equivalence class of a diagram $(p, q)$ with respect to the above relation is called a morphism and is denoted by

$$
\varphi=\{X \stackrel{p}{\Longleftarrow} Z \stackrel{q}{\longrightarrow} Y\}: X \longrightarrow Y .
$$

Given two morphisms $\varphi=\{X \stackrel{p}{\Longleftarrow} Z \stackrel{q}{\longrightarrow} Y\}: X \longrightarrow Y$ and $\psi=\left\{Y \stackrel{p^{\prime}}{\longleftarrow} Z^{\prime} \stackrel{q^{\prime}}{\longrightarrow} W\right\}: Y \longrightarrow W$, we write a commutative diagram: 


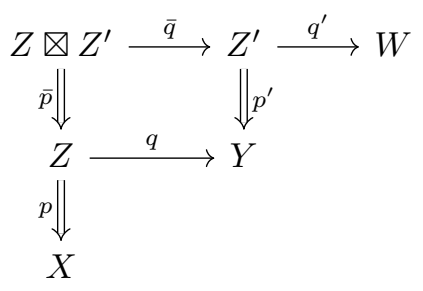

in which $Z \otimes Z^{\prime}=\left\{\left(z, z^{\prime}\right) \in Z \times Z^{\prime} ; q(z)=p^{\prime}\left(z^{\prime}\right)\right\}, \bar{p}\left(z, z^{\prime}\right)=z$ and $\bar{q}\left(z, z^{\prime}\right)=z^{\prime}$. Then, we define the composite of $\varphi$ and $\psi$ to be a morphism $\psi \circ \varphi=\left\{X \stackrel{p \circ \bar{p}}{\Longleftarrow} Z \otimes Z^{\prime} \stackrel{q^{\prime} \circ \bar{q}}{\longrightarrow} W\right\}: X \longrightarrow W \quad(\bar{p}$ is Vietoris being the pullback of $\left.p^{\prime}\right)$. It is easy to check that the above definition is independent of the choice of representatives. The law of composition allows to regard the collection of morphisms on spaces as a category $\mathcal{M}$. The category of usual single-valued maps regarded as morphisms of the form $\{X \stackrel{i d}{\Longleftarrow} X \longrightarrow Y\} \quad$ is a subcategory of $\mathcal{M}$. Górniewicz and Granas [9] established that the Čech homology functor extends to the category $\mathcal{M}$.

One easily see that morphisms can be treated as multivalued maps. We define an image of a point as the set $\varphi(x)=q\left(p^{-1}(x)\right)$ and it is obviously independent of the choice of representatives. We say that a morphism determines a multivalued map $\varphi$. Such a map $\varphi$ is upper-semicontinuous (see, e.g., [19] for a detailed but easy proof). A broad class of multivalued maps can be viewed in this way. A multivalued map $\varphi: X \multimap Y$ is acyclic provided it is u.s.c. and the image of each point $\varphi(x)$ is an acyclic compact set.

Proposition 2.4. A multivalued map $\varphi: X \multimap Y$ is determined by a morphism if and only if it is a composition of acyclic maps.

Proof. Every acyclic map is determined by a morphism of the form

$$
\varphi=\left\{X \stackrel{\pi_{1}}{\longleftarrow} \Gamma_{\varphi} \stackrel{\pi_{2}}{\longrightarrow} Y\right\}
$$

where $\Gamma_{\varphi}$ is the graph of $\varphi, \Gamma_{\varphi}=\{(x, y) \in X \times Y ; y \in \varphi(x)\}$ and $\pi_{1}, \pi_{2}$ are projections. Therefore, a composition of acyclic maps is determined by a composition of the above morphisms.

Now, given any morphism $\varphi=\{X \stackrel{p}{\Longleftarrow} Z \stackrel{q}{\longrightarrow} Y\}$, it is sufficient to notice that a map $\psi: X \rightarrow Z, \psi(x)=p^{-1}(x)$ is acyclic and $\varphi$ is a composition $\varphi=q \circ \psi$.

Remark 2.5. In [7], a broader class of admissible maps was considered. Those were maps admitting selections which were determined by morphisms.

Example. A multivalued map may be determined by different morphisms. Consider an acyclic map $\widetilde{\varphi}: S^{n} \rightarrow S^{n}, \widetilde{\varphi}(x)=\left\{y \in S^{n} ;\|y-x\| \leq \frac{3}{2}\right\}$, where $S^{n}=\left\{x \in \mathbb{R}^{n} ;\|x\|=1\right\}$.

It is determined by a morphism $\varphi=\left\{S^{n} \stackrel{\pi_{1}}{\Longleftarrow} \Gamma_{\widetilde{\varphi}} \stackrel{\pi_{2}}{\longleftrightarrow} S^{n}\right\}$. Therefore, the square $\varphi^{2}=\varphi \circ \varphi$ determines a map $\widetilde{\psi}: S^{n} \rightarrow S^{n}, \widetilde{\psi}(x)=S^{n}$. But 
$\widetilde{\psi}$ is also determined by any morphism of the form $\psi^{2}$,

$$
\psi=\left\{S^{n} \stackrel{\pi_{1}}{\Longleftarrow} \Gamma_{\varphi} \stackrel{f \circ \pi_{2}}{\longrightarrow} S^{n}\right\},
$$

where $f: S^{n} \rightarrow S^{n}$ is a map of degree $k$.

It is easily seen that $\varphi_{*}: H_{*}\left(S^{n}\right) \rightarrow H_{*}\left(S^{n}\right)$ is an identity isomorphism and so is $\varphi_{*}^{2}$. But $\psi_{*}$ is not an identity. Therefore, $\varphi^{2}$ and $\psi^{2}$ are different morphisms.

\section{Homotopy of morphisms}

There are various ways of defining a homotopy relation in the category $\mathcal{M}$. We have chosen the one which seems to be the most straightforward and has been used by some authors (see, e.g., $[1,4,7]$ ), though it was not obvious whether it was an equivalence relation.

Definition 3.1. Two diagrams

$$
X \stackrel{p}{\longleftarrow} Z \stackrel{q}{\longrightarrow} Y \text { and } X \stackrel{p^{\prime}}{\longleftarrow} Z^{\prime} \stackrel{q^{\prime}}{\longrightarrow} Y
$$

are homotopic if there is a diagram $X \times[0,1] \stackrel{\bar{p}}{\Longleftarrow} \bar{Z} \stackrel{\bar{q}}{\longrightarrow} Y$ and maps $f_{0}, f_{1}$ such that the following diagram commutes:

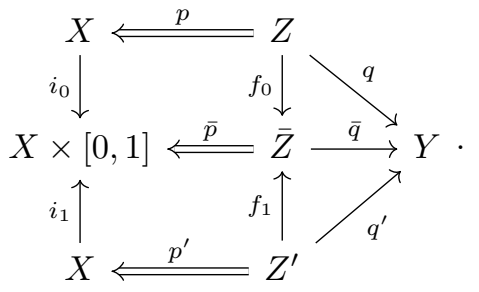

Here $i_{0}(x)=(x, 0), i_{1}(x)=(x, 1)$.

Proposition 3.2. The homotopy relation is an equivalence relation in the set of diagrams from $X$ to $Y$.

Proof. The only non-obvious property is the transitivity. Assume we have two commutative diagrams:
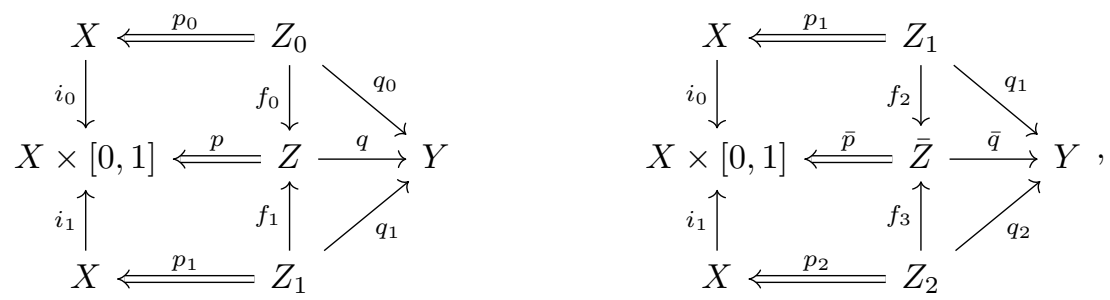

where $i_{k}(x)=(x, k)$. 

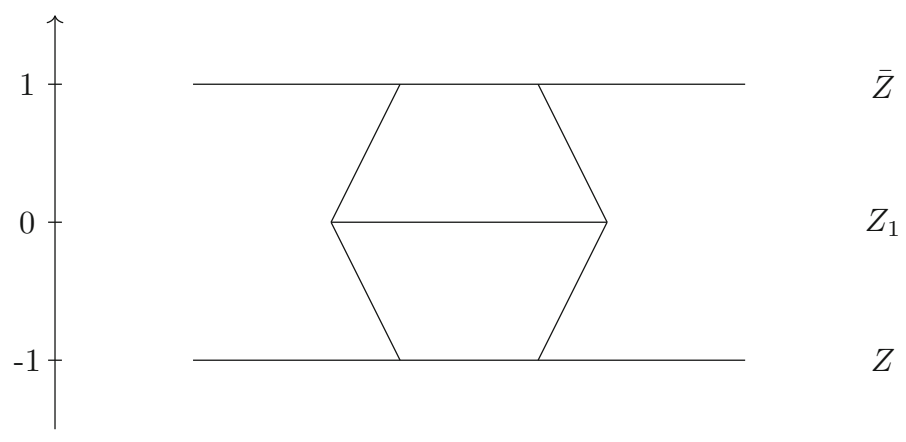

Figure 1. The space $K$

We have the mapping cylinders:

$$
\begin{gathered}
Z_{f_{1}}=Z_{1} \times[-1,0] \cup Z / \sim,(x,-1) \sim y \quad \text { iff } y=f_{1}(x), x \in Z_{1}, y \in Z ; \\
Z_{f_{2}}=Z_{1} \times[0,1] \cup \bar{Z} / \sim, \quad(x, 1) \sim y \quad \text { iff } y=f_{2}(x), x \in Z_{1}, y \in \bar{Z} .
\end{gathered}
$$
in Fig. 1.

Now, we define a space $K=Z_{f_{1}} \cup_{Z_{1}} Z_{f_{2}}$ which can be seen schematically

Define a map $\tilde{p}: K \Longrightarrow X \times[-1,1]$ by a formula

$$
\tilde{p}([x])=\left\{\begin{array}{ll}
\left(p_{1}(x), t\right) & \text { for }[x]=[(x, t)],-1 \leq t \leq 1 \\
\left(\pi_{1}(p(x)),-1\right) & \text { for } x \in Z \\
\left(\pi_{1}(\bar{p}(x)), 1\right) & \text { for } x \in \bar{Z}
\end{array},\right.
$$

and a map $\tilde{q}: K \longrightarrow Y$ by

$$
\tilde{q}([x])=\left\{\begin{array}{l}
q_{1}(x) \text { for }[x]=[(x, t)], x \in Z_{1} \\
q(x) \text { for } x \in Z \\
\bar{q}(x) \text { for } x \in \bar{Z}
\end{array} .\right.
$$

It follows immediately from the definition of $K$ that $\tilde{p}, \tilde{q}$ are correctly defined continuous maps. Moreover, $\tilde{p}$ is a Vietoris map since the preimages of points are exactly the same as for $p_{1}$ whenever $-1<t<1$, and for $t=1, t=-1$ the maps $\pi_{1} \circ p$ and $\pi_{1} \circ \bar{p}$, which are Vietoris maps, respectively.

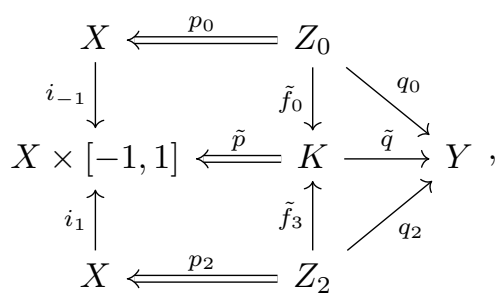

where $\tilde{f}_{0}(x)=j \circ f_{0}(x), \tilde{f}_{3}(x)=\bar{j} \circ f_{3}(x)$, with inclusions $j: Z \hookrightarrow K$, $\bar{j}: \bar{Z} \hookrightarrow K$.

Proposition 3.3. Assume that there exists a map $f: Z \rightarrow Z^{\prime}$ such that the following diagram commutes 


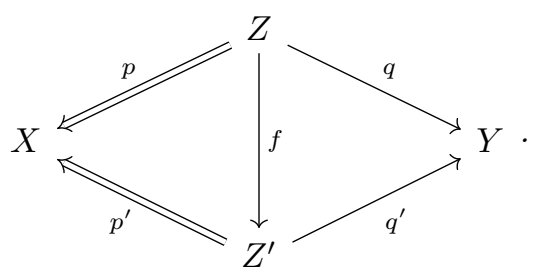

Then, the diagrams $X \stackrel{p}{=} Z \stackrel{q}{\longrightarrow} Y, \quad X \stackrel{p^{\prime}}{=} Z^{\prime} \stackrel{q^{\prime}}{\longrightarrow} Y \quad$ are homotopic.

Proof. It is sufficient to write a commutative diagram

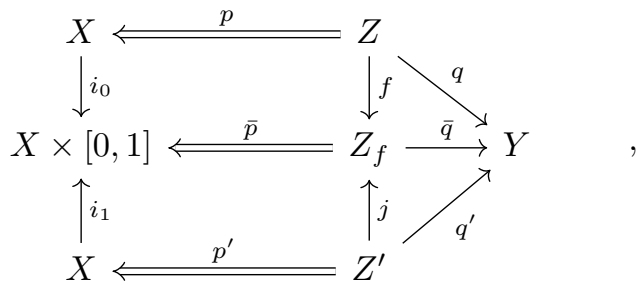

where the maps are defined by formulas:

$$
\begin{aligned}
& \bar{p}([x])=\left\{\begin{array}{l}
(p(x), t) \text { for }[x]=[(x, t)], x \in Z, t \in[0,1], \\
p^{\prime}(x) \quad \text { for } x \in Z^{\prime}
\end{array},\right. \\
& \bar{q}([x])=\left\{\begin{array}{l}
q(x) \text { for }[x]=[(x, t)], x \in Z, t \in[0,1], \\
q^{\prime}(x) \text { for } x \in Z^{\prime}
\end{array}\right.
\end{aligned}
$$

$\bar{f}(x)=[f(x)], j(x)=x$. It is easily seen that $\bar{p}, \bar{q}$ are well defined.

Definition 3.4. Two morphisms

$$
\varphi=\{X \stackrel{p}{\longleftarrow} Z \stackrel{q}{\longrightarrow} Y\} \text { and } \psi=\left\{X \stackrel{p^{\prime}}{\longleftarrow} Z^{\prime} \stackrel{q^{\prime}}{\longrightarrow} Y\right\}
$$

are homotopic if there is a morphism $\quad \chi=\{X \times[0,1] \stackrel{\bar{p}}{\longleftarrow} \bar{Z} \stackrel{\bar{q}}{\longrightarrow} Y\}$ and maps $f_{0}, f_{1}$ such that the following diagram commutes:

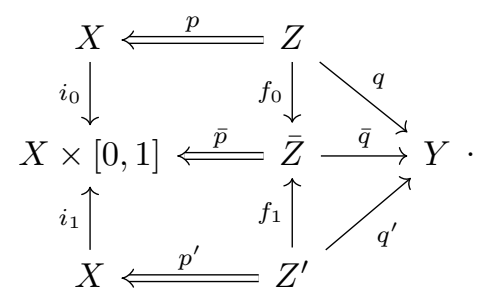

Here $i_{0}(x)=(x, 0), i_{1}(x)=(x, 1)$.

The following proposition is an immediate consequence of Propositions 3.2 and 3.3:

Proposition 3.5. The definition 3.4 does not depend on the choice of representatives. 
The following property is an immediate consequence of the definition of homotopy:

Proposition 3.6. Homotopic morphisms induce the same homology homomorphism.

Given three spaces $X, Y, Z$ and maps $\alpha: X \rightarrow Z, \beta: Y \rightarrow Z$ we denote by $X \underset{\alpha, \beta}{\otimes} Y$ their fiber-product $X \underset{\alpha, \beta}{\otimes} Y=\{(x, y) \in X \times Y ; \alpha(x)=\beta(y)\}$.

Proposition 3.7. Compositions of homotopic morphisms are also homotopic.

Proof. The proof is a diagram game. First, assume that

$$
\varphi_{0}=\left\{X \stackrel{p_{0}}{\Longleftarrow} Z_{0} \stackrel{q_{0}}{\longrightarrow} Y\right\}, \text { and } \varphi_{1}=\left\{X \stackrel{p_{1}}{\Longleftarrow} Z_{1} \stackrel{q_{1}}{\longrightarrow} Y\right\}
$$

are homotopic and let $\psi=\{W \stackrel{p}{\Longleftarrow} Z \stackrel{q}{\longrightarrow} X\}$.

Define a morphism $\widetilde{\psi}=\{W \times[0,1] \stackrel{\tilde{p}}{\longleftarrow} \widetilde{Z} \stackrel{\tilde{q}}{\longrightarrow} X \times[0,1]\}$, where $\widetilde{Z}=Z \times[0,1], \tilde{p}(x, t)=(p(x), t)$ and $\tilde{q}(x, t)=(q(x), t)$. We can write the following commutative diagram

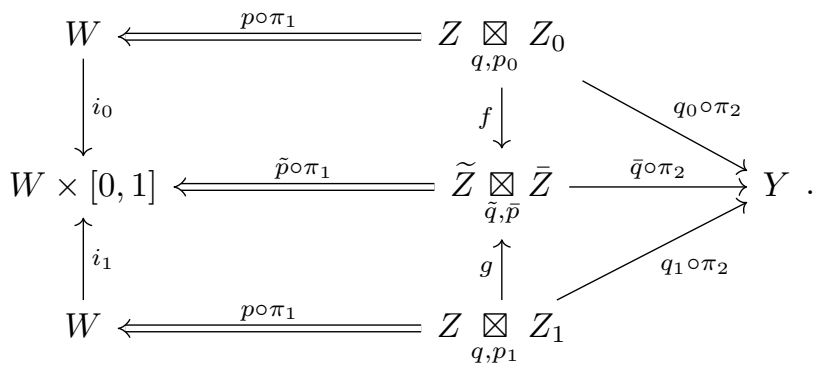

Here $f(x, y)=\left((x, 0), f_{0}(y)\right), g(x, y)=\left((x, 1), f_{1}(y)\right)$, where $f_{i}$ are the maps from the diagram defining a homotopy between $\varphi_{0}$ and $\varphi_{1}$ (see Def. 3.4), and $\pi_{1}(x, y)=x, \pi_{2}(x, y)=y$. One easily checks that the above diagram satisfies the conditions of 3.1 and thus the morphisms $\varphi_{0} \circ \psi$ and $\varphi_{1} \circ \psi$ are homotopic.

Now let $\varphi_{0}, \varphi_{1}$ be as above and $\psi=\{Y \stackrel{p}{\Longleftarrow} Z \stackrel{q}{\longrightarrow} W\}$. Consider the following diagram:

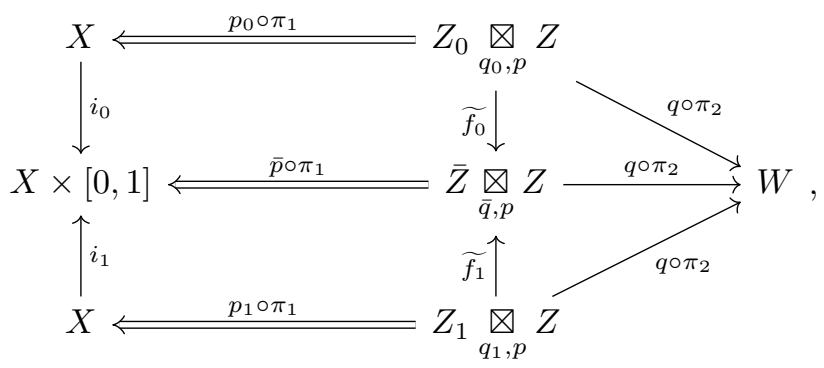

where $\pi_{i}$ are projections and $\widetilde{f}_{i}(x, y)=\left(f_{i}(x), y\right)$. The above diagram defines a homotopy joining morphisms $\psi \circ \varphi_{0}$ and $\psi \circ \varphi_{1}$. Now we use the transitivity property of homotopy to end the proof. 


\section{Acyclic maps and homotopy of morphisms}

As the Example 2 shows, different morphisms may determine the same multivalued map. In view of 3.6 these morphisms may even be not homotopic in general. The reason for it was the fact that the determined map in 2 was not acyclic.

Proposition 4.1. If $\varphi: X \multimap Y$ is an acyclic map then every two morphisms which determine a selection of $\varphi$, must be homotopic.

Proof. Consider the graph $\Gamma_{\varphi}=\{(x, y) \in X \times Y ; y \in \varphi(x)\}$ and maps $p_{\varphi}(x, y)=x, q_{\varphi}(x, y)=y$. Then $p_{\varphi}: \Gamma_{\varphi} \rightarrow X$ is a Vietoris map. Assume that $\psi=\{X \stackrel{p}{\Longleftarrow} Z \stackrel{q}{\longrightarrow} Y\} \quad$ determines a selection of $\varphi$ (i.e. for each $x \in X$ we have an inclusion $\psi(x) \subset \varphi(x))$. Then we have a commutative diagram

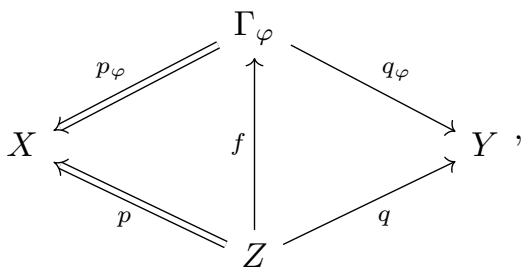

where $f(x)=(p(x), q(x))$. The assertion follows from 3.3.

Corollary 4.2. If two morphisms $\varphi_{0}, \varphi_{1}: X \rightarrow Y$ have a common acyclic support (i.e., an acyclic map such that $\varphi_{0}, \varphi_{1}$ determine its selections) then, the induced homology homomorphisms are the same.

Proposition 4.3. There exist non-homotopic single-valued maps which are homotopic as morphisms.

Proof. It is enough to consider a space $X$ which is acyclic but not contractible. Then, the identity map $i d: X \rightarrow X$ is not homotopic to any constant map $y_{0}(x)=y_{0}$. But they are homotopic as morphisms since they are selections of the acyclic map $\varphi: X \multimap X, \varphi(x)=X$.

\section{A local coincidence index for morphisms}

In [5], an index theory for a broad class of multi-valued maps including admissible maps as well some maps with non-connected images of points has been constructed (comp. [7], Ch. IV). It has been proved that such maps induce arbitrarily fine chain approximations on nerves of coverings in the sense of [22] (see [5, Thm 4.3]). Regarding a representative of a morphism $\varphi=\{X \stackrel{p}{\longleftarrow} Y \stackrel{q}{\longrightarrow} X\} \quad$ as a sequence of two acyclic maps $p^{-1}$ and $q$ there are chain maps induced by it. If $X$ is a compact polyhedron and $U$ is a polyhedral open subset of $X$ such that $x \notin q\left(p^{-1}(x)\right)$ for $x \in \partial U$, an index of $(p, q)$ can be defined to be a 'local Lefschetz number' (comp. 
$[5,22])$. It has been proved to be independent of the choice of approximation and representatives (since they are all homotopic). Therefore, the index $i(X, \varphi, U)$ of a morphism $\varphi$ is defined (see [5, Def. 5.6]) to be a rational number (more exactly, it is an element of the coefficient field). The definition can be extended in quite a routine way to the case of a morphism $\varphi=\{X \stackrel{p}{\longleftarrow} Y \stackrel{q}{\longrightarrow} X\}$, where $X$ is a metric ANR, $\overline{\varphi(X)}$ compact and $U \subset X$ an open set with $x \notin \varphi(x)$ for $x \in \partial U$ (equivalently $p(x) \neq q(x)$ for $\left.x \in p^{-1}(\partial U)\right)$. The detailed proofs can be found in [5].

Let $(K, \tau)$ be a compact polyhedron with a fixed triangulation $\tau$. Its $n$-th barycentric subdivision is denoted by $\tau^{n}$. A subset $U \subset K$ is polyhedral provided there is an integer $l$ such that $\tau^{l}$ induces a triangulation of the closure $\bar{U}$ of $U$ in $K$.

Let $l$ be a natural number and $F$ a field. We denote by $C_{*}\left(K, \tau^{l}\right)$ the oriented chain complex $C_{*}\left(K, \tau^{l} ; F\right)$. The carrier of $c \in C_{*}(K, \tau)(\operatorname{carr} c)$ is the smallest polyhedral subset $X \subset K$ such that $c \in C_{*}(X, \tau)$. By $b$ : $C_{*}(K, \tau) \rightarrow C_{*}\left(K, \tau^{l}\right)$ we denote the subdivision chain mapping which maps each chain into its $l$-th barycentric subdivision.

By $\chi: C_{*}\left(K, \tau^{l}\right) \rightarrow C_{*}(K, \tau)$, we denote a chain mapping which is induced by a simplicial approximation of the identity map id $:\left(K, \tau^{l}\right) \rightarrow$ $(K, \tau)$. It is known that $b$ and $\chi$ are chain homotopy inverses to each other. In the context of approximations the notion of a closed star is useful. Let $B \subset K$ be a subset. The $k$-th closed star of $B$ in $(K, \tau)$ is defined inductively (simplices are closed here):

$$
\begin{aligned}
& S t^{1}(B, \tau)=S t(B, \tau):=\bigcup\{\sigma \in \tau ; \sigma \cap B \neq \emptyset\}, \\
& S t^{k}(B, \tau):=S t\left(S t^{k-1}(B, \tau), \tau\right) .
\end{aligned}
$$

Let $\Phi:(K, \tau) \multimap(L, \mu)$ be an u.s.c. multivalued mapping.

Definition 5.1. Let $k$ and $l$ be two natural numbers.

A chain mapping $\varphi: C_{*}\left(K, \tau^{l}\right) \rightarrow C_{*}\left(L, \mu^{k}\right)$ is called an $(n, k)$ approximation of $\Phi$ provided the following conditions holds: for each simplex $\sigma \in \tau^{l}$, there exists a point $y(\sigma) \in K$ such that

$$
\sigma \subset S t^{n}\left(y(\sigma), \tau^{l}\right), \operatorname{carr} \varphi \sigma \subset S t^{n}\left(\Phi(y(\sigma)), \mu^{k}\right)
$$

Definition 5.2. A graded set

$$
A(\Phi)=\left\{A(\Phi)_{j}\right\}_{j \in \mathbb{N}}
$$

where $A(\Phi)_{j} \subset \operatorname{hom}\left(C_{*}\left(K, \tau^{j}\right), C_{*}\left(L, \mu^{j}\right)\right)$ is called an approximation system ( $A$-system) for $\Phi$ provided there is an integer $n=n(A)$ such that:

(i) If $\varphi \in A(\Phi)_{j}$ then $\varphi=\varphi_{1} \circ b$, where $\varphi_{1}$ is an $(n, j)$-approximation of $\Phi$.

(ii) For every $j \in \mathbb{N}$, there exists $j_{1} \in \mathbb{N}$ such that for $m \geq l \geq j_{1}$ and for all $\varphi=\varphi_{1} \circ b \in A(\Phi)_{l}$ and $\psi=\psi_{1} \circ b \in A(\Phi)_{m}$ the diagram 


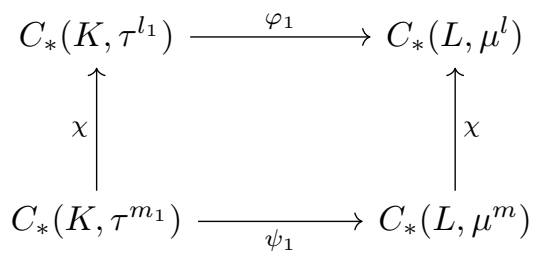

with $m_{1} \geq l_{1}$ is homotopy commutative with a chain homotopy $D$ satisfying the following smallness condition:

- For any simplex $\sigma \in \tau^{m_{1}}$, there exists a point $z(\sigma) \in K$ such that

$$
\sigma \subset S t^{n}\left(z(\sigma), \tau^{j}\right), \operatorname{carr} D \sigma \subset S t^{n}\left(\Phi(z(\sigma)), \mu^{j}\right) .
$$

Definition 5.3. Let $\Phi_{1}, \Phi_{2}: K \multimap L$ be u.s.c. maps and let $H: K \times[0,1] \multimap L$ be an u.s.c. homotopy joining $\Phi_{1}$ and $\Phi_{2}$. Let $A\left(\Phi_{1}\right)$ and $A\left(\Phi_{2}\right)$ be $A$-systems for $\Phi_{1}$ and $\Phi_{2}$, respectively. They are $H$-homotopic provided there is an integer $m \in \mathbb{N}$ such that the following condition holds:

(I) For every $j \in \mathbb{N}$, there is $j_{1} \in \mathbb{N}$ such that for any $l \geq j_{1}$ there are $\varphi=\varphi_{1} \circ b \in A\left(\Phi_{1}\right)_{l}$ and $\psi=\psi_{1} \circ b \in A\left(\Phi_{2}\right)_{l}$ such that $\varphi_{1}, \psi_{1}$ : $C_{*}\left(K, \tau^{l_{1}}\right) \rightarrow C_{*}\left(L, \mu^{l}\right)$ are chain homotopic with an $H$-small homotopy $D$, i.e.:

(i) For $\sigma \in \tau^{l_{1}}$ there is a point $d(\sigma) \in K$ such that

$$
\sigma \subset S t^{m}\left(d(\sigma), \tau^{j}\right), \operatorname{carr} D \sigma \subset S t^{m}\left(H(d(\sigma) \times[0,1]), \mu^{j}\right) .
$$

One can define an index for an $A$-system as follows: Let $K$ be a compact polyhedron with a given triangulation $\tau$ and let $U$ be a polyhedral open subset of $K$ (i.e., $U$ is open and $\bar{U}$ is a subpolyhedron of $K$ ). Let $\Phi: \bar{U} \multimap K$ be such that $x \notin \Phi(x)$ for $x \in \partial U$, and let $A=A(\Phi)$ be an $A$-system for $\Phi$. Denote by $p_{U}: C_{*}\left(K, \tau^{k}\right) \rightarrow C_{*}\left(\bar{U}, \tau^{k}\right)$ the natural linear projection and let $\varphi \in A(\Phi)_{k}$. We define a 'local Lefschetz number'

$$
\lambda\left(p_{U} \circ \varphi\right):=\sum_{i=0}^{\operatorname{dim} K}(-1)^{i} \operatorname{tr}\left(p_{U} \circ \varphi\right)_{i} .
$$

It has been proved in [22] that there exists sufficiently large $k_{0}$ such that the above defined number does not depend on the choice of $\varphi \in A(\Phi)_{k}, k \geq k_{0}$.

Thus, we can define the index properly.

Definition 5.4. $i_{A}(K, \Phi, U):=\lambda\left(p_{U} \circ \varphi\right)$ for $\varphi \in A(\Phi)_{k}, k \geq k_{0}$.

Proposition 5.5. Properties of index:

(1) (Additivity) Let $U_{1}, U_{2}$ be open, disjoint and polyhedral subsets of $U$ and $\Phi: \bar{U} \rightarrow K$ an u.s.c. mapping such that $\operatorname{Fix}(\Phi) \subset U_{1} \cup U_{2}$. If $A(\Phi)$ is an A-system for $\Phi$ then

$$
i_{A}(K, \Phi, U)=i_{A}\left(K, \Phi, U_{1}\right)+i_{A}\left(K, \Phi, U_{2}\right)
$$


(2) (Excision) Let $U_{1} \subset U$ be two open and polyhedral subsets of $K$ and $\operatorname{Fix}(\Phi) \subset U_{1}$. Then,

$$
i_{A}(K, \Phi, U)=i_{A}\left(K, \Phi, U_{1}\right) .
$$

(3) (Homotopy invariance) Let $H: \bar{U} \times[0,1] \rightarrow K$ be an u.s.c. homotopy such that $x \notin H(x, t)$ for $x \in \partial U$ and $t \in[0,1]$. Let $A_{0}, A_{1}$ be $H$ homotopic A-systems for $H_{0}=H(\cdot, 0)$ and $H_{1}=H(\cdot, 1)$, respectively. Then,

$$
i_{A_{0}}\left(K, H_{0}, U\right)=i_{A_{1}}\left(K, H_{1}, U\right) .
$$

(4) (Commutativity) Let $W \subset K$ be open and let $\Phi_{1}: K \rightarrow L, \Phi_{2}: L \rightarrow K$ be u.s.c. maps.

Assume that $x \notin \Phi_{2} \circ \Phi_{1}(x)$ for $x \in \partial W$ and $y \notin \Phi_{1} \circ \Phi_{2}(y)$ for $y \in \partial\left(\Phi_{2}^{-1}(W)\right)$. Assume further that if $y \in \operatorname{Fix}\left(\Phi_{1} \circ \Phi_{2}\right) \backslash \Phi_{2}^{-1}(W)$ then $\left.\Phi_{2}(y) \cap \operatorname{Fix}\left(\Phi_{2} \circ \Phi_{1}\right)\right|_{\bar{W}}=\emptyset$. Then for any A-systems $A_{1}=A\left(\Phi_{1}\right)$, $A_{2}=A\left(\Phi_{2}\right)$

$$
i_{A_{1} \circ A_{2}}\left(L, \Phi_{1} \circ \Phi_{2}, \Phi_{2}^{-1}(W)\right)=i_{A_{2} \circ A_{1}}\left(K, \Phi_{2} \circ \Phi_{1}, W\right) .
$$

(5) (Mod-p property) Let the coefficient field $F=\mathbb{Z}_{p}, p$ prime. Let $W \subset K$ be open and $\Phi: K \rightarrow K$ an u.s.c. map such that $x \notin \Phi^{p}(x)$ for $x \in \partial W$. Assume that if $y \in \operatorname{Fix}\left(\Phi^{p}\right) \backslash W$ then $\left.\Phi^{k}(y) \cap \operatorname{Fix}\left(\Phi^{p}\right)\right|_{\bar{W}}=\emptyset$ for $k<p$. Then

$$
i_{A}(K, \Phi, W)=i_{A^{p}}\left(K, \Phi^{p}, W\right) .
$$

Remark 5.6. Because of Prop. 5.5(2), one can also define the index $i_{A}(K, \Phi, V)$, when $V$ is open and not polyhedral, if $\Phi: L \rightarrow K, V \subset L \subset K$, and $L$ is a subpolyhedron of $K$. Then one puts

$$
i_{A}(K, \Phi, V):=i_{A}(K, \Phi, U),
$$

where $U \subset V$ is polyhedral and $\left.\operatorname{Fix}(\Phi)\right|_{\bar{V}} \subset U$.

The existence of an $A$-system for maps determined by morphisms was proved in fact in [5], where more general maps were considered. Recall that we can associate an abstract simplicial complex with a locally finite covering $\alpha$ of a given space $X$, called its' nerve $N(\alpha)$. Simplices of $N(\alpha)$ are the nonempty intersections of the sets $V_{i} \in \alpha$. We denote the support of a simplex $s \in N(\alpha)$ as the union of the forming sets (vertices). Similarly we define the star of a set as the union of all simplices intersecting it. We denote by $N^{n}(\alpha)$ the $n$ dimensional skeleton of the nerve. Let $X, Y$ be compact spaces. By $\operatorname{Cov} X$, $\operatorname{Cov} Y$ we denote the sets of finite open coverings of these spaces, respectively.

Definition 5.7. Let $\alpha, \bar{\alpha} \in \operatorname{Cov} X, \beta, \bar{\beta} \in \operatorname{Cov} Y$ and let $\Phi: X \multimap Y$.

A chain map $\varphi: C_{*}\left(N^{n}(\bar{\alpha})\right) \rightarrow C_{*}\left(N^{n}(\bar{\beta})\right)$ is called an $(\alpha, \beta)$ approximation of $\Phi$ provided: for every simplex $s \in N^{n}(\bar{\alpha})$ there exists a point $p(s) \in X$ such that

$$
\operatorname{supp} s \subset S t(p(s), \alpha), \quad \operatorname{supp} \varphi s \subset S t(\Phi(p(s)), \beta) \text {. }
$$


The following basic approximation result is a special case of Theorem 4.3 in $[5]$ :

Theorem 5.8. Let $\Phi: X \multimap Y$ be an acyclic-valued u.s.c. map of compact spaces, and $\alpha \in \operatorname{Cov} X, \beta \in \operatorname{Cov} Y$. Then for every $n \in \mathbb{N}$, there exist: a refinement $\bar{\alpha}$ of $\alpha$ and an $(\alpha, \beta)$ - approximation $\varphi: C_{*}\left(N^{n}(\bar{\alpha})\right) \rightarrow C_{*}\left(N^{n}(\beta)\right)$ of $\Phi$.

Recall that given a morphism $\varphi=\{X \stackrel{p}{\Longleftarrow} Z \stackrel{q}{\longrightarrow} Y\} \quad$, the map $p^{-1}: X \multimap Z$ is u.s.c. and acyclic, and Theorem 5.8 applies. Thus, we can formulate

Proposition 5.9. If $(K, \tau),(L, \mu)$ are compact polyhedra and

$$
\varphi=\{K \stackrel{p}{\Longleftarrow} Z \stackrel{q}{\longrightarrow} L\}
$$

is a morphism, then the compositions of chain approximations of $p^{-1}$ and $q$ form an A-system.

Proof. Let us recall that given a triangulation $\tau$, we can associate an open covering $\alpha(\tau)$ by the sets $\operatorname{st}(v, \tau)=\operatorname{Int}(\operatorname{St}(v, \tau))$, where $v$ are vertices of the triangulation. The simplicial maps $\theta:(K, \tau) \rightarrow N(\alpha(\tau)), \lambda: N(\alpha(\tau)) \rightarrow$ $(K, \tau)$ defined on vertices by $\theta(v):=\operatorname{st}(v, \tau)$, and $\lambda(s t(v, \tau)):=v$, define a natural simplicial isomorphism. The same is true for $(L, \mu)$. Considering barycentric subdivisions of the triangulation $\tau$ we obtain arbitrarily fine refinements of $\alpha(\tau))$. It has been detailly proved that the compositions of sufficiently close chain approximations: $\psi_{2}$ of the acyclic map $p^{-1}$ and $\psi_{1}$ of the single-valued map $q$ are chain homotopic (see [5, Prop. 4.8 and 4.14], [8, Prop. 50.30 and 50.39]).

Therefore, the compositions of the form $\lambda \circ \psi_{1} \circ \psi_{2} \circ \theta \circ b$ form an $A$ system for the given morphism $\varphi$. The map $b$ is the standard subdivision map (see [23]) and $\lambda, \theta$ are the above isomorphisms for barycentric subdivisions $\tau^{k}, \mu^{k}$.

Let now $U$ be an open polyhedral subset of a compact polyhedron $(K, \tau)$ (i.e. $\bar{U}$ is a subpolyhedron) and let $\varphi: \bar{U} \rightarrow K$ be a morphism such that $x \notin \varphi(x)$ (equivalently $p(y) \neq q(y)$ for $y \in p^{-1}(\partial U)$ for the representatives).

Then, we define

$$
i(K, \varphi, U):=i_{A}(K, \varphi, U) .
$$

By localization property, we do not need to assume that $U$ is polyhedral.

Now let $X$ be a compact metric ANR, $U \subset X$ open and let $\varphi: \bar{U} \rightarrow$ $X$ be a morphism such that Fix $\varphi \cap \partial U=\emptyset$. Then, there exists a finite polyhedron $K \varepsilon$-dominating $X$ with $\varepsilon>0$ smaller than the distance of Fix $\varphi$ from $\partial U$ (see [3, p. 41]). Denoting the maps $r: K \rightarrow X, s: X \rightarrow K$ $\left(r \circ s=i d_{X}, d(s \circ r(x), x)<\varepsilon\right)$, we define the index as follows:

$$
i(X, \varphi, U):=i\left(K, s \circ \varphi \circ r, r^{-1}(U)\right) .
$$

Independent of the choice of $K, r, s$ is detailly proved in [5], and [8, Sect. IV.52]. 
The next step is to extend the definition of the index to the case of arbitrary ANRs. By the Arens-Eells embedding Theorem (see, e.g., [8, Thm 1.6]) we can assume that $X$ is a closed subset of a normed space $E$. Since $X$ is an ANR, there is an open subset $V \subset E$ and a retraction $r: V \rightarrow X$. Consider a compact morphism $\varphi=\{\bar{U} \stackrel{p}{\Longleftarrow} Z \stackrel{q}{\longrightarrow} X\}$ such that $\overline{q(Z)}$ is compact, and $p(z) \neq q(z)$ for $p(z) \in \partial U$. Denoting by $i: X \rightarrow V$ the inclusion map we define a morphism $\psi=i \circ \varphi \circ r: U^{\prime}=r^{-1}(\bar{U}) \rightarrow V$. One easily checks that $\operatorname{Fix} \psi \cap r^{-1}(\partial U)=\emptyset$. By the result of J. Girolo [6] there exists a compact ANR $K$ such that $\overline{\psi\left(U^{\prime}\right)} \subset K \subset V$. We let $W=K \cap r^{-1}(U)$ and then the index $i\left(K,\left.\psi\right|_{K}, W\right)$ is well defined as above.

Thus, we define

$$
i(X, \psi, U)=i\left(K,\left.\psi\right|_{K}, W\right) .
$$

Theorem 5.10. ([5, Prop. 7.5]) The index has the following properties:

1. Additivity. If $U_{1}, U_{2} \subset U$ are open and disjoint and if Fix $\left.\varphi\right|_{\bar{U}} \subset$ $U_{1} \cup U_{2}$ then $i(X, \varphi, U)=i\left(X, \varphi, U_{1}\right)+i\left(X, \varphi, U_{2}\right)$.

2. Homotopy invariance. Assume that the morphisms $\varphi, \psi: X \rightarrow X$ are homotopic by a homotopy such that for its representatives holds $x \notin q\left(p^{-1}(x, t)\right)$ whenever $x \in \partial U$. Then $i(X, \varphi, U)=i(X, \psi, U)$.

3. Normalization. If $\Lambda\left(\varphi_{*}\right)$ denotes the (generalized) Lefschetz number of the induced homology homomorphism then $i(X, \varphi, X)=\Lambda\left(\varphi_{*}\right)$.

4. Commutativity. Let $W \subset X$ be open and $\varphi: X \rightarrow Y, \psi: Y \rightarrow X$ be morphisms. Assume that $x \notin \psi \circ \varphi(x)$ for $x \in \partial W$ and $y \notin \varphi \circ \psi(y)$ for $y \in \partial\left(\psi^{-1}(W)\right)$ and moreover $\left.\psi\left(\operatorname{Fix} \varphi \circ \psi-\psi^{-1}(W)\right) \cap \operatorname{Fix} \psi \circ \varphi\right|_{\bar{W}}=\emptyset$. Then $i(X, \psi \circ \varphi, W)=i\left(Y, \varphi \circ \psi, \psi^{-1}(W)\right)$.

Corollary 5.11. If $i(X, \varphi, U) \neq 0$ then the determined map has a fixed point in $U$ (or equivalently any representative $X \stackrel{p}{\Longleftarrow} Z \stackrel{q}{\longrightarrow} X$ has a coincidence in $\left.p^{-1}(U)\right)$.

Corollary 5.12. If $Y \subset X$ is an $A N R$ and $\varphi(\bar{U}) \subset Y$, then

$$
i(X, \varphi, U)=i(Y, \varphi, U \cap Y) .
$$

The first corollary is a consequence of additivity and the last one of commutativity (see. Thm 5.10).

Now, we prove a generalization of the well-known nonlinear alternative (comp. [11, II.6, Thm 5.2]) from which one can derive many fixed point or coincidence results. We omit the corollaries since they are routine (comp. $[4,7,11])$.

Theorem 5.13. Let $C$ be a convex subset of a normed space $E$ and $U$ an open set in $C$ containing 0 . Then for any morphism

$$
\varphi=\{C \stackrel{p}{\Longleftarrow} Z \stackrel{q}{\longrightarrow} C\}: C \longrightarrow C
$$

one of the following conditions is satisfied: 
(i) $\varphi$ has a fixed point in $\bar{U}$,

(ii) for some $x \in \partial U$ and $0<t<1$ there is $y \in p^{-1}(x)$ such that $x=t \cdot q(y)$.

Proof. Consider the following commutative diagram

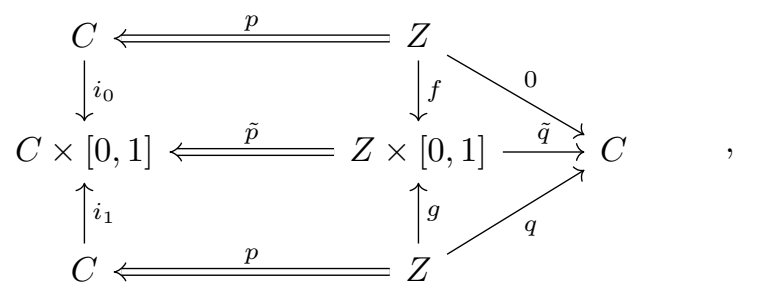

where $\bar{p}(x, t)=(p(x), t), \bar{q}(x, t)=t \cdot q(x), 0(x)=0, f(x)=(x, 0)$ and $g(x)=(x, 1)$. Denoting by $\psi$ the morphism defined by $(p, 0)$ we observe that $i(C, \psi, U)=1$ since $0 \in U$ and it is a constant map $x \mapsto 0$. If $\varphi$ has a fixed point in $U$ then we are done. If not and if moreover $(i i)$ is not satisfied that means exactly that assumptions of homotopy invariance (Thm 5.10) are fulfilled. Therefore, $i(C, \varphi, U)=1$ and $\varphi$ has a fixed point in $U$ by Cor. 5.11.

Remark 5.14. We assumed in this section that the considered morphisms determined compact maps, but one can extend all the results to some noncompact maps. We touched the example of compact attraction maps in [5]. The classes of condensing morphisms mentioned in [15] (comp. [24]) may be also included into the theory.

Remark 5.15. The index is a priori a rational number but there are no examples where it is not an integer. Moreover, in [2], it was proved that in the case of acyclic maps of ENR-s, the index is an integer and the theory is unique.

\section{Extending morphisms from a sphere}

In this short section, we prove one extremely simple geometric result which analog for single-valued maps is an exercise (see, e.g., [11, Thm II.5.1]). We say a morphism is nullhomotopic provided it is homotopic to a morphism $\varphi=\left\{X \stackrel{i d}{\longleftarrow} X \stackrel{y_{0}}{\longleftrightarrow} Y\right\}, \quad$ where $y_{0}(x)=y_{0}$ is any constant map.

Proposition 6.1. A morphism $\varphi=\left\{S^{n} \stackrel{p}{\Longleftarrow} Z \stackrel{q}{\longrightarrow} Y\right\} \quad$ is nullhomotopic if and only if $\varphi$ is extendable to a morphism $\Phi: D^{n+1} \rightarrow Y$ in the unit disc.

Proof. Assume $\varphi$ is nullhomotopic, i.e., we have a diagram

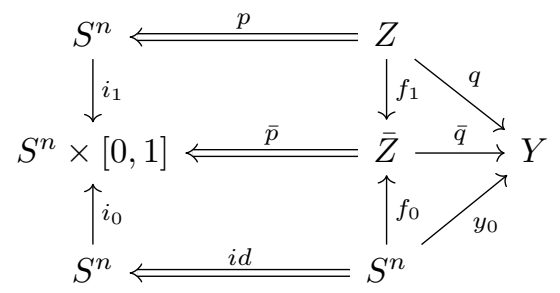




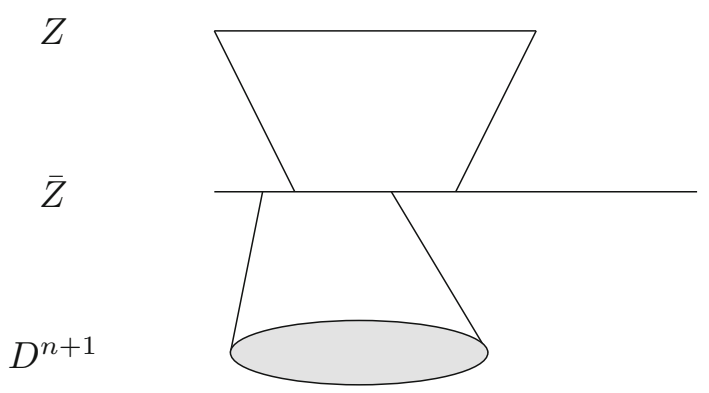

Figure 2. A sketch of $K$

We define a morphism $\Phi: D^{n+1} \rightarrow Y$. Let $K$ be a space obtained from the disjoint sum of three spaces $Z \times[0,1], \bar{Z}, S^{n} \times[0,1] \cup D^{n+1} \times\{0\}$ by the following relations: if $x \in Z$ then $(x, 0) \sim f_{1}(x)$, and if $x \in S^{n}$ then $(x, 1) \sim f_{0}(x)$ (see Fig. 2 for a sketch).

Define a map $\tilde{p}: K \Longrightarrow D^{n+1}$ by the formula

$$
\tilde{p}([x])= \begin{cases}\frac{1}{4}(3+t) p(x) & \text { if }[x]=[(x, t)], x \in Z \\ g(\bar{p}(x)) & \text { if } x \in \bar{Z} \\ \frac{1}{4}(1+t) x & \text { if }[x]=[(x, t)], x \in S^{n}, \\ \frac{1}{4} x & \text { if } x \in D^{n+1}\end{cases}
$$

where $g: S^{n} \times[0,1] \rightarrow D^{n+1}, g(x, t)=\frac{1}{4}(2+t) x$.

A map $\tilde{q}: K \rightarrow Y$ is defined by the formula

$$
\tilde{q}([x])=\left\{\begin{array}{l}
q(x) \text { if }[x]=[(x, t)], x \in Z \\
\bar{q}(x) \text { if } x \in \bar{Z} \\
y_{0} \text { in other case }
\end{array} .\right.
$$

It is easily seen that $p$ is a Vietoris map, and moreover

$$
p^{-1}\left(S^{n}\right)=Z \times\{1\} \approx Z .
$$

Hence a morphism $\Phi=\left\{D^{n+1} \stackrel{\tilde{p}}{\longleftarrow} K \stackrel{\tilde{q}}{\longrightarrow} Y\right\} \quad$ is an extension of $\varphi$.

Conversely, assume we have a morphism $\left\{D^{n+1} \stackrel{\bar{p}}{\Longleftarrow} \bar{Z} \stackrel{\bar{q}}{\longrightarrow} Y\right\}$. There is a map $g: S^{n} \times[0,1] \rightarrow D^{n+1}$ given by

$$
g(x, t)=\left\{\begin{array}{ll}
0 & \text { if } 0 \leq t \leq \frac{1}{2} \\
(2 t-1) x & \text { if } \frac{1}{2} \leq t \leq 1
\end{array} .\right.
$$

Define a space $K=\left\{(x, t, y, z) \in S^{n} \times[0,1] \times D^{n+1} \times \bar{Z} ; y=g(x, t)=\bar{p}(z)\right\}$. Then we have a commutative diagram

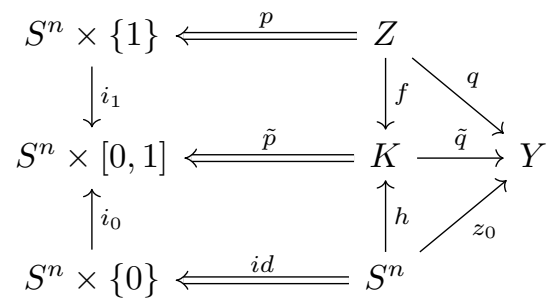


Here $Z=\bar{p}^{-1}\left(S^{n} \times\{1\}\right), \tilde{p}(x, t, y, z)=(x, t), \tilde{q}(x, t, y, z)=\bar{q}(z), f(x)=$ $=(\bar{p}(x), 1, \bar{p}(x), x), h(x)=\left(x, 0,0, y_{0}\right)$, where $y_{0}$ is any point in $\bar{p}^{-1}(0)$ and $z_{0}=q\left(y_{0}\right)$, and $p, q$ are restrictions of $\bar{p}, \bar{q}$. The diagram proves that $\left\{S^{n} \stackrel{p}{\longleftarrow} Z \stackrel{q}{\longrightarrow} Y\right\} \quad$ is a morphism homotopic to a constant map.

Combining Propositions 6.1 together with 4.3, we obtain:

Corollary 6.2. If $Y$ is an acyclic space with $\pi_{n}(Y) \neq 0$ then there exists a single-valued map $f: S^{n} \rightarrow Y$ such that $f$ is not extendable to $D^{n+1}$ as a map but it is extendable as a morphism (there is a multivalued extension).

The results of this section suggest that one can extend theorems concerning essential maps of $[11, \mathrm{Ch}$. II]. A version of this approach can be found in $[16-18]$.

\section{Acknowledgements}

We are grateful to Anonymous Reviewer for very careful reading of the manuscript and many constructive remarks.

Open Access. This article is licensed under a Creative Commons Attribution 4.0 International License, which permits use, sharing, adaptation, distribution and reproduction in any medium or format, as long as you give appropriate credit to the original author(s) and the source, provide a link to the Creative Commons licence, and indicate if changes were made. The images or other third party material in this article are included in the article's Creative Commons licence, unless indicated otherwise in a credit line to the material. If material is not included in the article's Creative Commons licence and your intended use is not permitted by statutory regulation or exceeds the permitted use, you will need to obtain permission directly from the copyright holder. To view a copy of this licence, visit http:// creativecommons.org/licenses/by/4.0/.

Publisher's Note Springer Nature remains neutral with regard to jurisdictional claims in published maps and institutional affiliations.

\section{References}

[1] Andres, J.: On the coexistence of irreducible orbits of coincidences for multivalued admissible maps on the circle via Nielsen theory Topol. Appl. 221, 596-609 (2017)

[2] Bielawski, R.: The fixed point index for acyclic maps on ENR-s Bull. Pol. Acad. Sci. 35, 487-499 (1987)

[3] Brown, R.F.: The Lefschetz Fixed Point Theorem Scott. Foresman and Co. Glenview III, London (1971)

[4] Dzedzej, Z.: Degree of coincidence in locally convex spaces Coll. Math. Soc. Janos Bolyai 23, Topology, Budapest (1978), 377-395

[5] Dzedzej, Z.: Fixed point index theory for a class of nonacyclic multivalued maps Dissert. Math. 253, 1-58 (1985) 
[6] Girolo, J.: Approximating compact sets in normed linear spaces Pacific. J. Math. 98, 81-89 (1982)

[7] Górniewicz, L.: Homological methods in fixed point theory of mulivalued maps Dissert. Math. 129, 1-71 (1976)

[8] Górniewicz, L.: Topological Fixed Point Theory of Multivalued Mappings, 2nd edn. Springer, Dortrech (2006)

[9] Górniewicz, L., Granas, A.: Some general theorems in coincidence theory I. J. Math. Pures et Appl. 60, 361-373 (1981)

[10] Granas, A.: Sur la methode de continuité de Poincaré C. R. Acad. Sci Paris 282, 978-985 (1976)

[11] Granas, A., Dugundji, J.: Fixed Point Theory. Springer, New York (2003)

[12] Hara, Y., Moriwaki, Y.: The degree of multivalued maps from manifolds to spheres. J. Fixed Point Theory Appl. 11, 253-259 (2012)

[13] Kryszewski, W.: Topological and approximation methods of degree theory of set-valued maps Dissert. Math. 336, 1-101 (1994)

[14] Kucharski, Z.: A coincidence index Bull. Acad. Polon. Sci. 24, 245-252 (1976)

[15] Nussbaum, R.: Generalizing the fixed point index Math. Ann. 228, 259-278 (1977)

[16] O'Regan, D.: Coincidence theory for compact morphisms Fixed Point Theory Appl. 19, (2017)

[17] O'Regan, D.: Coincidence for morphisms based on compactness conditions on countable sets. Appl. Math. Comput. 339, 59-62 (2018)

[18] O'Regan, D.: Coincidence for morphisms based on compactness principles. J. Nonlinear Sci. Appl. 11, 1096-1098 (2018)

[19] Segiet, W.: Local coincidence index for morphisms Bull. Acad. Polon. Sci. 30, 261-267 (1982)

[20] Shitanda, Y.: The degree of set-valued mappings from ANR spaces to homology spheres Math. J. Okayama Univ. 59, 27-40 (2017)

[21] Shitanda, Y.: A generalization of antipodal point theorems for set-valued mappings. Hokkaido Math. J. 20(3), 217-238 (2010)

[22] Siegberg, H.W., Skordev, G.: Fixed point index and chain approximations. Pacific J. Math. 102, 455-486 (1982)

[23] Spanier, E.H.: Algebraic Topology. McGraw-Hill, New York (1966)

[24] Violette, D., Fournier, G.: A fixed point index for compositions of acyclic multivalued maps in Banach space (in French) Ann. Sci. Math. Québec 22(2), 225-244 (1998)

Zdzisław Dzedzej and Tomasz Gzella

Faculty of Technical Physics and Applied Mathematics

Gdańsk University of Technology

ul. Narutowicza 11/12

80-233 Gdańsk

Poland

e-mail: zdzedzej@mif.pg.gda.pl;

tomasz.gzella@pg.edu.pl

Accepted: September 18, 2020. 